\title{
Aperture-Angle and Hausdorff-Approximation of Convex Figures
}

\author{
Hee-Kap Ahn • Sang Won Bae · Otfried Cheong • \\ Joachim Gudmundsson
}

Received: 16 February 2007 / Revised: 12 September 2007 /

Published online: 10 October 2007

(C) Springer Science+Business Media, LLC 2007

\begin{abstract}
The aperture angle $\alpha(x, Q)$ of a point $x \notin Q$ in the plane with respect to a convex polygon $Q$ is the angle of the smallest cone with apex $x$ that contains $Q$. The aperture angle approximation error of a compact convex set $C$ in the plane with respect to an inscribed convex polygon $Q \subset C$ is the minimum aperture angle of any $x \in C \backslash Q$ with respect to $Q$. We show that for any compact convex set $C$ in the plane and any $k>2$, there is an inscribed convex $k$-gon $Q \subset C$ with aperture angle approximation error $\left(1-\frac{2}{k+1}\right) \pi$. This bound is optimal, and settles a conjecture by Fekete from the early $1990 \mathrm{~s}$.

The same proof technique can be used to prove a conjecture by Brass: If a polygon $P$ admits no approximation by a sub- $k$-gon (the convex hull of $k$ vertices of $P$ ) with Hausdorff distance $\sigma$, but all subpolygons of $P$ (the convex hull of some vertices of $P$ ) admit such an approximation, then $P$ is a $(k+1)$-gon. This implies the follow-
\end{abstract}

This research was supported by the Korea Research Foundation Grant funded by the Korean Government (MOEHRD) (KRF-2006-311-D00763).

NICTA is funded through the Australian Government's Backing Australia's Ability initiative, in part through the Australian Research Council.

H.-K. Ahn

Department of Computer Science and Engineering, POSTECH, Pohang, Korea

e-mail: heekap@postech.ac.kr

S.W. Bae $\cdot$ O. Cheong $(\varangle)$

Division of Computer Science, Korea Advanced Institute of Science and Technology,

Daejon, Korea

e-mail: otfried@tclab.kaist.ac.kr

S.W. Bae

e-mail: swbae@tclab.kaist.ac.kr

J. Gudmundsson

National ICT Australia Ltd, Sydney, Australia

e-mail: joachim.gudmundsson@nicta.com.au 
ing result: For any $k>2$ and any convex polygon $P$ of perimeter at most 1 there is a sub-k-gon $Q$ of $P$ such that the Hausdorff-distance of $P$ and $Q$ is at most $\frac{1}{k+1} \sin \frac{\pi}{k+1}$.

Keywords Hausdorff approximation · Aperture angle $\cdot$ Convex figure $\cdot$ Subpolygon

\section{Introduction}

Let $Q$ be a compact set in the plane, and let $x$ be a point outside $Q$. The aperture angle $\alpha(x, Q)$ of $x$ with respect to $Q$ is the angle of the smallest cone with apex $x$ that contains $Q$ (that is, its boundary consists of two rays emanating from $x$ tangent to $Q$ ). The aperture angle plays a role in various applications related to sensing, and has been studied in a number of papers. Bose et al. [1], for instance, consider two disjoint convex polygons $P$ and $Q$ in the plane, and give algorithms

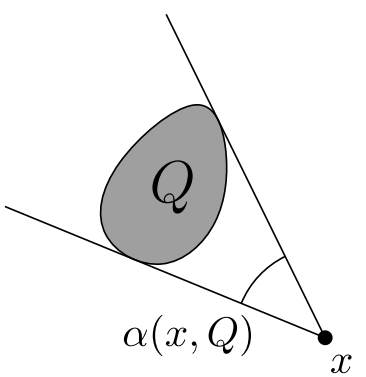
to compute the maximum aperture angle and the minimum aperture angle with respect to $Q$ when $x$ is allowed to vary in $P$. Hoffmann et al. [7] introduce the angle hull of a connected region $Q$ inside a simple polygon $P$, consisting of all those points $x \in P$ with $\alpha(x, Q)$ at least a given angle. They give bounds on the length of the boundary of the angle hull, and apply this to the problem of exploring an unknown environment. Cheong and van Oostrum [5] give bounds on the complexity of the angle hull of a convex polygon in a polygonal environment, and apply this to the problem of motion planning under directional uncertainty.

We consider the problem of placing $k$ sensors or transmitters in a compact convex room $C$. A point in $C$ is covered perfectly if it lies inside the convex hull $Q \subset C$ of the sensors. However, if $C$ has more than $k$ vertices or even a smooth boundary, we must have $Q \subsetneq C$, and it is not possible to achieve this for all points of $C$. For points $x \in C \backslash Q$, we would like to maximize the coverage by the sensors, and measure this using the aperture angle $\alpha(x, Q)$. For given $C$ and $Q \subset C$, let us denote the worst coverage as

$$
\alpha(C, Q):=\min _{x \in C} \alpha(x, Q),
$$

where we set $\alpha(x, Q)=\pi$ for $x \in Q$. Since $C$ is compact and $\alpha(x, Q)$ is continuous, the minimum is indeed attained in $C$ and this is well-defined. We are looking for the best placement of $k$ sensors, so we seek to maximize $\alpha(C, Q)$ over all convex $k$-gons inscribed in $C$. Let us denote this quantity as $\alpha(C, k)$, defined formally as

$$
\alpha(C, k):=\max _{Q \in \mathcal{C}_{k}(C)} \alpha(C, Q),
$$

where $\mathcal{C}_{k}(C)$ is the family of convex $k$-gons inscribed to $C$. In other words, we study the approximation of convex sets by inscribed $k$-gons with respect to the "apertureangle distance." This distance measure is attractive as it is naturally scale-invariant, without needing to be normalized by some global property of $C$ (such as its perimeter or area). We are now interested in the following question: Given $k$, what aperture 
angle can we guarantee for any possible compact convex $C$ ? In other words, we ask for the following quantity

$$
\alpha(k):=\inf _{C \in \mathcal{C}} \alpha(C, k)=\inf _{C \in \mathcal{C}} \max _{Q \in \mathcal{C}_{k}(C)} \min _{x \in C} \alpha(x, Q),
$$

where $\mathcal{C}$ is the family of compact convex figures in the plane. This question was first asked by Fekete in 1990, and circulated at several open problem sessions in the early 1990s [6]. An upper bound for $\alpha(k)$ is given by the regular convex $(k+1)$-gon $P_{k+1}$. Since any $k$-gon $Q$ inscribed in $P_{k+1}$ must "miss" a vertex of $P_{k+1}$, we have $\alpha\left(P_{k+1}, Q\right) \leq(1-2 /(k+1)) \pi$ (the interior angle at each vertex of $\left.P_{k+1}\right)$. For a lower bound, we can walk around the boundary of a given $C$ and place a vertex of $Q$ whenever the tangent direction has changed by $2 \pi / k$. It is easy to see that this achieves $\alpha(C, Q) \geq(1-2 / k) \pi$, and so we have $\alpha(k) \geq(1-2 / k) \pi$.

A discrepancy between the two bounds remained, and Fekete conjectured that the upper bound is correct:

Conjecture 1 For any $k \geq 2$, the smallest value of $\alpha(k)$ is achieved by the regular $(k+1)$-gon, and we therefore have

$$
\alpha(k)=\left(1-\frac{2}{k+1}\right) \pi .
$$

Fekete already showed that his conjecture holds for $k=2$ and $k=3$, and experiments in Jenkner's Master thesis [8] indicate that it should hold for general $k$. The problem was also published by Brass and Lassak [3], and it appears again as Problem 5 in Sect. 11.3 of Brass et al.'s encyclopedic collection of research problems in discrete geometry [4], with a short proof of the case $k \in\{2,3\}$.

If $P$ is a convex polygon, then it is known that $\alpha(P, k)$ can be attained by an inscribed subpolygon. Here, a subpolygon of $P$ is the convex hull of a subset of $P$ 's vertices. This provides for an interesting similarity between Conjecture 1 and the following conjecture by Brass on Hausdorff approximation by subpolygons:

Conjecture 2 Let $\mathcal{P}$ be a family of convex polygons in $\mathbb{R}^{2}$ that is closed under taking subpolygons. If $\mathcal{P}$ has an element that is hardest to approximate by its $k$-vertex subpolygons with respect to the Hausdorff metric, then one can also find a $(k+1)$-gon in $\mathcal{P}$ with this property.

Conjecture 2 appears as Conjecture 5 in Sect. 11.5 of Brass et al. [4]. It was first suggested by Brass in 2000 [2].

In this paper, we prove both Conjecture 1 and 2. Our proof relies on a combinatorial analysis of worst-approximable polygons in Sect. 3. Here, a polygon $P$ is worst-approximable if every proper subpolygon $R$ of $P$ admits a better approximation by $k$-vertex subpolygons than $P$ does. Our analysis in Sect. 3 makes no use of the geometry of the problem, and applies to any approximation measure that is monotone in the sense that "growing" $Q \subset P$ cannot increase the approximation error (a formal definition of monotonicity is in Sect. 2). 
In Sect. 4 we bring back in the geometry of the aperture angle problem, and prove that a convex polygon that is worst-approximable under aperture-angle approximation is in fact a $(k+1)$-gon. This implies a positive answer to Conjecture 1 for the case of polygons. The case of arbitrary compact convex sets is then proven using a limit argument.

Similarly, we study the geometry of the Hausdorff problem in Sect. 5 and prove that any polygon that is worst-approximable under Hausdorff approximation is again a $(k+1)$-gon.

As an application of this result, we finally consider the family $\mathcal{P}$ of convex polygons with unit perimeter. We show that the $(k+1)$-gon $P$ in $\mathcal{P}$ that admits the worst approximation by a $k$-vertex subpolygon is the regular $(k+1)$-gon. Together with our result on Conjecture 2, this implies that for every convex polygon $P$ with unit perimeter and every $k>2$ there is a $k$-vertex subpolygon $Q$ of $P$ such that the Hausdorff distance between $P$ and $Q$ is at most $\frac{1}{k+1} \sin \frac{\pi}{k+1}$. This result is the "subpolygon version" of a result by Popov, who had proven that any convex figure $C$ of perimeter one admits an inscribed $k$-gon $Q$ with Hausdorff-distance at most $\frac{1}{2 k} \sin \frac{\pi}{k}$ [10]. Popov's result is not known to be tight. Popov conjectured that the regular $(k+1)$-gon is the worst case $[9,10]$. Our result shows that the equivalent statement is true for approximation by subpolygons.

\section{Preliminaries}

Let $P$ be a convex $n$-gon and let $V$ be the set of its vertices. For three vertices $p, u, q \in V$, we say that $u$ lies between $p$ and $q$ and write $p \preceq u \preceq q$ if a counterclockwise traversal of $P$ starting at $p$ encounters $u$ before $q$ (and $u$ is allowed to coincide with $p$ or $q$ ). If we do not allow $u$ to coincide with $p$, we write $p \prec u \preceq q$, see Fig. 1a.

For any subset $V^{\prime} \subset V$, the convex hull of $V^{\prime}$ is a subpolygon of $P$. A subpolygon $Q$ is proper if $Q \neq P$. We are interested in subpolygons $Q$ of $P$ that closely approximate $P$. Let $\varphi(P, Q) \geq 0$ denote the approximation error of $P$ with respect to a subpolygon $Q$ of $P$. We will consider two different error functions: the apertureangle and the Hausdorff-distance (to be defined below). Let $\mathcal{C}_{k}(P)$ denote the set of convex subpolygons of $P$ with at most $k$ vertices and let $\varphi_{k}(P)$ denote the smallest error that can be achieved by a polygon in $\mathcal{C}_{k}(P)$, that is,

$$
\varphi_{k}(P):=\min _{Q \in \mathcal{C}_{k}(P)} \varphi(P, Q) .
$$

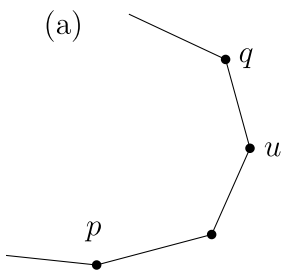

(b)

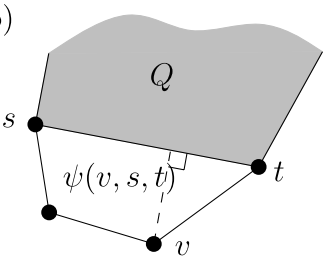

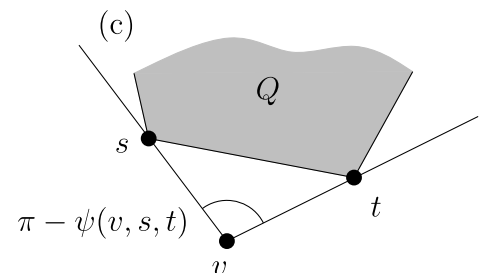

Fig. 1 a $u$ lies between $p$ and $q$, that is, $p \prec u \prec q$. b Illustrating the Hausdorff distance, and $\mathbf{c}$ the aperture-angle 
Clearly $\varphi_{k}(P)=0$ if $P$ has at most $k$ vertices. We require that $\varphi(P, Q)$ can be expressed as

$$
\varphi(P, Q)=\max _{v \in V} \varphi(v, Q),
$$

where $\varphi(v, Q)$ is zero if $v$ is a vertex of $Q$, and is otherwise of the form

$$
\varphi(v, Q)=\psi(v, s, t),
$$

where $s$ and $t$ are the two consecutive vertices of $Q$ with $s \prec v \prec t$. Furthermore, we require the function $\psi$ to be monotone, that is, if $s \preceq s^{\prime} \prec v \prec t^{\prime} \preceq t$, then $\psi\left(v, s^{\prime}, t^{\prime}\right) \leq \psi(v, s, t)$.

If we set $\psi(v, s, t)$ to be the distance of $v$ from the segment $s t$, then $\varphi(P, Q)$ is the Hausdorff-distance between $P$ and $Q$, as shown in Fig. 1b.

If we set $\psi(v, s, t)=\pi-\angle s v t$, then $\pi-\varphi(P, Q)$ is the smallest aperture angle of any vertex of $P$ with respect to $Q$, see Fig. 1c. Since it is easy to see that $\alpha(x, Q)$ is minimized within $P$ at the vertices of $P$, this implies $\varphi(P, Q)=\pi-\alpha(P, Q)$. Note that we use the complement of the angle since we want to minimize the error, but maximize the angle.

\section{Properties of Worst-Approximable Polygons}

Let us call a convex polygon $P$ worst-approximable if for every proper subpolygon $Q$ of $P$ we have $\varphi_{k}(Q)<\varphi_{k}(P)$. In this section we study the nature of worstapproximable polygons. Our arguments are purely combinatorial, using only the monotonicity of $\psi$.

We start by introducing some basic concepts. A pair $(p, q) \in V^{2}$ is called a diagonal. For a given approximation error $\sigma>0$, a diagonal $(p, q)$ is called feasible if for every $v \in V$ with $p \prec v \prec q$ we have $\psi(v, p, q) \leq \sigma$. By monotonicity of $\psi$, if $(p, q)$ is feasible, then so is any $\left(p^{\prime}, q^{\prime}\right)$ with $p \preceq p^{\prime} \prec q^{\prime} \preceq q$. A feasible diagonal $(p, q)$ is called a chord if it is the feasible diagonal starting at $p$ of largest combinatorial length, where the combinatorial length of a diagonal $(p, q)$ is the number of edges on the polygon boundary from $p$ to $q$ (in counter-clockwise direction). In other words, $(p, q)$ is a chord if $q$ is the last vertex on $P$ (starting from $p$ ) such that $(p, q)$ is feasible.

The chord graph $\mathcal{G}$ is a directed graph with vertex set $V$, such that $(p, q)$ is an edge of $\mathcal{G}$ if $(p, q)$ is a chord. Clearly, every vertex of $\mathcal{G}$ has out-degree one.

Let $k>2$ and $\sigma>0$ be fixed, and assume that $P$ is a convex $n$-gon with $\varphi_{k}(P)>\sigma$, but such that for every proper subpolygon $R$ of $P$ we have $\varphi_{k}(R) \leq \sigma$. As before, let $V$ be the set of $P$ 's vertices, and let $\mathcal{G}$ be the chord graph of $P$ for the approximation error $\sigma$. For $u \in V$, let $P_{u}$ be the convex hull of $V \backslash\{u\}$, see Fig. 2a. By assumption, $P_{u}$ has a $k$-gon approximation $Q_{u}$ with error $\leq \sigma$. Without loss of generality, we can assume that $Q_{u}$ consists of $k-1$ chords and of an edge st, where $s \prec u \prec t$, as illustrated in Fig. 2b. For all $x \in V \backslash\{u\}$ with $s \prec x \prec t$ we have $\psi(x, s, t) \leq \sigma$, but $\psi(u, s, t)>\sigma$.

We call $s t$ the base of $Q_{u}$, and note that $Q_{u}$ is completely determined by $t$, since the other $k-1$ vertices can be found by following $k-1$ arcs from $t$ in the chord 
(a)

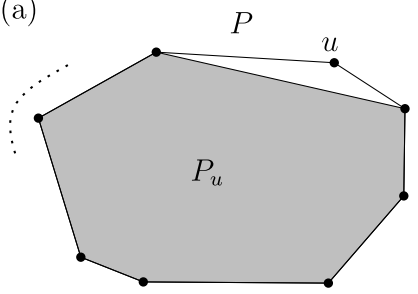

(b)

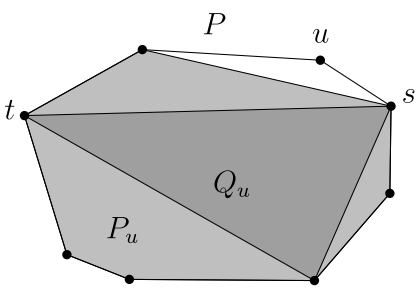

Fig. 2 a Illustrating $P$ and $P_{u}$, and $\mathbf{b} Q_{u}$ with base $s t$

graph. Let $s: V \mapsto V$ and $t: V \mapsto V$ be functions mapping $u$ to the clockwise $(s)$ and counter-clockwise $(t)$ endpoints of the base of $Q_{u}$.

Lemma 1 The function $t$ is a bijection.

Proof Assume that there exist two subpolygons $Q_{u}$ and $Q_{v}, u \neq v$, of $P$ that both have base $s t$. Since $s t$ is the base of $Q_{u}$ and $s \prec v \prec t$, we have $\psi(u, s, t)>\sigma$ and $\psi(v, s, t) \leq \sigma$. This, however, contradicts $\psi(v, s, t)>\sigma$ due to $s t$ being the base of $Q_{v}$. Thus, the base of each $Q_{u}$ is distinct. Since $Q_{u}$ is completely determined by $t(u)$, the function $t$ is an injection, and therefore a bijection.

For a base $s t$, let the witness $w(s t)$ of $s t$ be the unique vertex with $s \prec w(s t) \prec t$ with $\psi(w(s t), s, t)>\sigma$. The witness of the base of $Q_{u}$ is of course $u$. There are thus $n$ bases in total, and their witnesses are all distinct.

Lemma 2 It is impossible for two bases st and $s^{\prime} t^{\prime}$ to be nested, that is, to realize the order

$$
s \preceq s^{\prime} \prec t^{\prime} \preceq t
$$

Proof Assume the opposite, that is, $s \preceq s^{\prime} \prec t^{\prime} \preceq t$. Since $w\left(s^{\prime} t^{\prime}\right) \neq w(s t)$ we have $\psi\left(w\left(s^{\prime} t^{\prime}\right), s, t\right) \leq \sigma$. By monotonicity of $\psi$ this implies $\psi\left(w\left(s^{\prime} t^{\prime}\right), s^{\prime}, t^{\prime}\right) \leq \sigma$, a contradiction.

Next we study the nature of the chord graph and prove that it is surprisingly symmetric. We denote the vertices $V$ of $P$ as $v_{0}, v_{1}, \ldots, v_{n-1}$ in counter-clockwise order. Throughout the paper, arithmetic on indices of $v$ will be modulo $n$.

Lemma 3 Every vertex in the chord graph has in-degree and out-degree one. There is an integer $m>1$ such that every chord is of the form $\left(v_{i}, v_{i+m}\right)$.

Proof From the definition of chord graphs it immediately follows that the out-degree of each vertex is one. Since the number of arcs in the chord graph is $n$, it suffices to show that no vertex has in-degree greater than one. Assume that $q$ is a vertex with in-degree at least two, that is, there are chords $(p, q)$ and $\left(p^{\prime}, q\right)$, with $p \prec p^{\prime} \prec q$. Let $u=t^{-1}(p)$ and $u^{\prime}=t^{-1}\left(p^{\prime}\right)$, and so $t(u)=p$ and $t\left(u^{\prime}\right)=p^{\prime}$. Since we can find $s(u)$ from $t(u)$ by following $k-1$ arcs in the chord graph, $t(u)=t\left(u^{\prime}\right)$ implies 

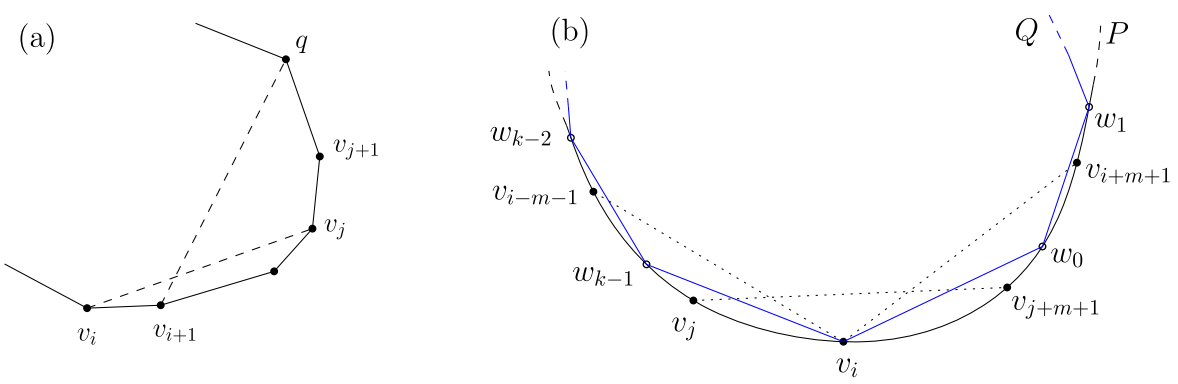

Fig. 3 a Illustrating the proof of Lemma 3, and $\mathbf{b}$ the proof of Lemma 5

$s(u)=s\left(u^{\prime}\right)$. Now we have $s\left(u^{\prime}\right) \preceq s(u) \preceq t(u) \prec t\left(u^{\prime}\right)$, a contradiction to Lemma 2 . It follows that no vertex of the chord graph has in-degree greater than one.

Next we consider two chords $\left(v_{i}, v_{j}\right)$ and $\left(v_{i+1}, q\right)$, as shown in Fig. 3a. If $q \neq v_{j+1}$ then there has to be a chord $\left(p, v_{j+1}\right)$ with $v_{i} \prec p \prec v_{i+1} \prec v_{j} \prec v_{j+1} \prec q$, a contradiction. This implies that two consecutive chords must have the same combinatorial length, and so all chords do.

We will use $m$ to denote the combinatorial length of all chords. For every $0 \leq i<n,\left(v_{i}, v_{i+m}\right)$ is the chord starting at $v_{i}$, and $\left(v_{i-m}, v_{i}\right)$ is the chord ending at $v_{i}$.

Recall that every $Q_{u}$ has $k-1$ chords and a base. Since the combinatorial length of every chord is $m$ by Lemma 3, it follows immediately that every base must have length $n-(k-1) m$ :

Corollary 1 Every base is of the form $\left(v_{i}, v_{i+n-(k-1) m}\right)$.

It turns out that we can prove even stronger properties about the bases.

Lemma 4 All bases have combinatorial length $m+1$, and so $n=k m+1$.

Proof By Corollary 1 all bases have combinatorial length $b:=n-(k-1) m$. Assume that $b \neq m+1$. Since a base must have larger combinatorial length than a chord, it suffices to consider the case when $b>m+1$. Consider two consecutive bases $\left(v_{0}, v_{b}\right)$ and $\left(v_{1}, v_{b+1}\right)$. Consider the diagonal $\left(v_{1}, v_{b}\right)$. Since $b>m+1$, this diagonal is not feasible, and so there is a vertex $w \in V$ with $v_{1} \prec w \prec v_{b}$ with $\psi\left(w, v_{1}, v_{b}\right)>\sigma$.

By monotonicity, this implies $\psi\left(w, v_{0}, v_{b}\right)>\sigma$, and $\psi\left(w, v_{1}, v_{b+1}\right)>\sigma$. However, $w\left(v_{0} v_{b}\right)$ is the only vertex in the range $v_{0} \prec x \prec v_{b}$ with $\psi\left(x, v_{0}, v_{b}\right)>\sigma$, and so $w=w\left(v_{0} v_{b}\right)$. Similarly, $w\left(v_{1} v_{b+1}\right)$ is the only vertex in the range $v_{1} \prec x \prec$ $v_{b+1}$ with $\psi\left(x, v_{1}, v_{b+1}\right)>\sigma$, and so $w=w\left(v_{1} v_{b+1}\right)$. It follows that $w\left(v_{0} v_{b}\right)=$ $w\left(v_{1} v_{b+1}\right)$, a contradiction since all witnesses are distinct.

Lemma 5 Let $v_{i} \in V$, and consider the two witnesses $w\left(v_{i-m-1} v_{i}\right)$ and $w\left(v_{i} v_{i+m+1}\right)$. If $n>k+1$ then at least one of the two witnesses lies in the range $s\left(v_{i}\right) \prec x \prec t\left(v_{i}\right)$.

Springer 
Proof Set $v_{j}:=s\left(v_{i}\right)$ and note that $v_{i-m-1} \prec v_{j} \prec v_{i} \prec v_{j+m+1} \prec v_{i+m+1}$, as shown in Fig. 3b. Consider the $k$ witnesses $w_{a}:=w\left(v_{i+a m} v_{i+a m+m+1}\right)$, where $0 \leq a \leq k-1$. Note that $w_{0}=w\left(v_{i} v_{i+m+1}\right)$, and $w_{k-1}=w\left(v_{i-m-1} v_{i}\right)$ since $v_{i-m-1}=v_{i+(k-1) m}$.

The proof is done by contradiction, thus assume $v_{i-m-1} \prec w_{k-1} \preceq v_{j}$ and $v_{j+m+1} \preceq w_{0} \prec v_{i+m+1}$. Now consider the subpolygon $R$ with the $k+1$ vertices $\left\{v_{i}, w_{0}, w_{1}, \ldots, w_{k-1}\right\}$. We will show that $\varphi_{k}(R)>\sigma$, which is a contradiction to the definition of $P$.

First, we observe that $v_{i+a m} \prec w_{a} \preceq v_{i+(a+1) m}$ and that $w_{k-1} \prec v_{i} \prec w_{0}$. This implies that the vertices of $R$ are $v_{i}, w_{0}, w_{1}, \ldots, w_{k-1}$ in this order. It remains to verify the approximation error.

From the definition of a witness we have $\psi\left(w_{a}, v_{i+a m}, v_{i+a m+m+1}\right)>\sigma$ and $w_{a-1} \preceq v_{i+a m} \prec w_{a} \prec v_{i+a m+m+1} \preceq w_{a+1}$, for $1 \leq a \leq k-2$. Putting these two observations together implies that $\psi\left(w_{a}, w_{a-1}, w_{a+1}\right)>\sigma$, since $\psi$ is a monotone function.

Similarly, since $\psi\left(w_{0}, v_{i}, v_{i+m+1}\right)>\sigma$ and $w_{0} \prec v_{i+m+1} \preceq w_{1}$, we have $\psi\left(w_{0}, v_{i}, w_{1}\right)>\sigma$.

Since $\psi\left(w_{k-1}, v_{i-m-1}, v_{i}\right)>\sigma$ and $w_{k-2} \preceq v_{i-m-1} \prec w_{k-1}$, we also have $\psi\left(w_{k-1}, w_{k-2}, v_{i}\right)>\sigma$.

Finally, since $v_{i}=w\left(v_{j} v_{j+m+1}\right)$, we have $\psi\left(v_{i}, v_{j}, v_{j+m+1}\right)>\sigma$. Since $w_{k-1} \preceq$ $v_{j} \prec v_{i} \prec v_{j+m+1} \preceq w_{0}$, we also have $\psi\left(v_{i}, w_{k-1}, w_{0}\right)>\sigma$.

Hence, for any subpolygon $Q$ of $R$ with $k$ vertices, the vertex $v$ of $R$ not in $Q$ has approximation error $\varphi(v, Q)>\sigma$, implying $\varphi(R, Q)>\sigma$.

\section{Aperture-Angle Approximation}

In order to proceed with our arguments, we need to get back into the geometry of the problem. In this section we consider the case of aperture angle approximation, that is, our error function is $\psi(v, s, t)=\pi-\angle s v t$ as illustrated in Fig. 1c.

For two points $p, q$ in the plane, let $H^{+}(p, q)$ be the half-plane to the right of the oriented line from $p$ to $q$. For any $0<\sigma<\pi$ we define

$$
D_{\sigma}(p, q):=\left\{x \in H^{+}(p, q) \mid \angle p x q \geq \pi-\sigma\right\} .
$$

The region $D_{\sigma}(p, q)$ is the intersection of a disk containing $p$ and $q$ on its boundary with $\mathrm{H}^{+}(p, q)$, see Fig. 4a. Its boundary consists of a circular arc with endpoints $p$ and $q$ and of the segment $p q$. For fixed $\sigma$, the regions $D_{\sigma}(p, q)$ for any pair $p, q$ are affinely similar - that is, one can be obtained from the other by a rigid motion and a scaling - and, in particular, the radius of the circular arc is proportional to the distance $d(p, q)$. If $0<\sigma<\pi / 2$, then the center of the circular arc lies to the left of the oriented line $p q$, and so the circular arc spans less than a semi-circle. We observe that $\psi(v, s, t) \leq \sigma$ if and only if $v \in D_{\sigma}(s, t)$ (note that $s \preceq v \preceq t$ implies that $\left.v \in H^{+}(s, t)\right)$.

We will need a simple geometric fact, which can be proven using elementary calculations, see Fig. 4b. 
(a)

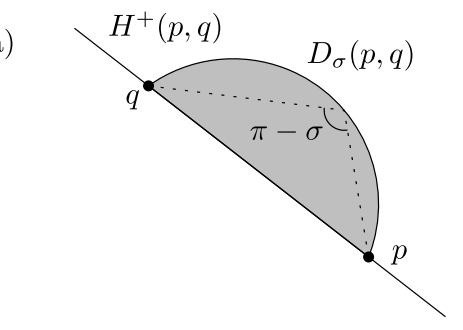

(b)

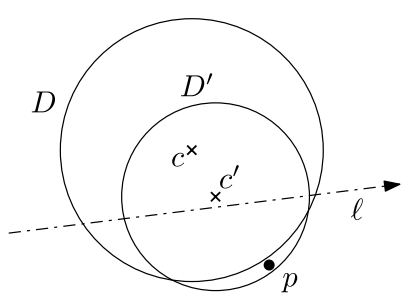

(c)

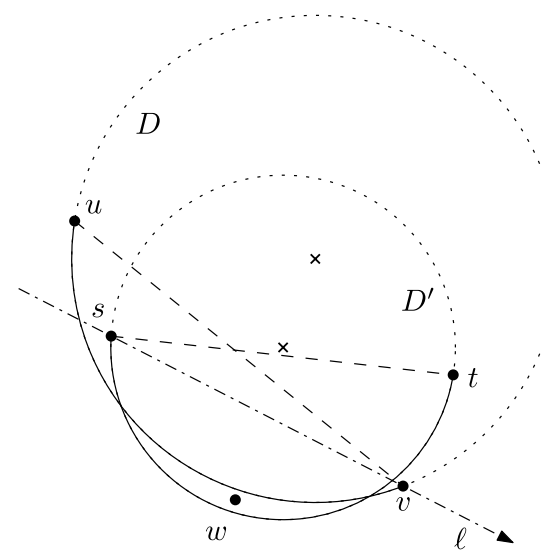

Fig. 4 a The region $D_{\sigma}(p, q)$. b The radius of $D^{\prime}$ is smaller than that of $D$. c $D$ and $D^{\prime}$ fulfill the assumptions of Lemma 6

Lemma 6 Consider two disks $D$ and $D^{\prime}$, whose centers lie to the left of an oriented line $\ell$. If $D^{\prime} \cap \ell$ is contained in $D \cap \ell$, but there is a point $p \in D^{\prime} \backslash D$ to the right of $\ell$, then the radius of $D^{\prime}$ is smaller than the radius of $D$.

Lemma 7 If $P$ is a convex $n$-gon that is worst-approximable with respect to the aperture angle and $k>2$ then $n=k+1$.

Proof Let $P$ be a worst-approximable convex $n$-gon with respect to the aperture angle, and assume that the statement of the lemma is false, that is, $n>k+1$. Let $\sigma:=$ $\max _{R} \varphi_{k}(R)$, where the maximum is taken over all proper subpolygons $R$ of $P$. Since $P$ is worst-approximable, we have $\varphi_{k}(P)>\sigma$, and since for every proper subpolygon $R$ of $P$ we have $\varphi_{k}(R) \leq \sigma$, the results of Sect. 3 apply. Since $\alpha(3)=\pi / 2$ [4], we have $\sigma<\varphi_{k}(P) \leq \pi / 2$.

Let now $v$ be a vertex of $V$ that maximizes the Euclidean distance $d(s(v), t(v))$. According to Lemma 5 there is an incident base, say $u v$, such that $u \prec s(v) \prec$ $w(u v) \prec v$ (the other case being symmetric). We let $w:=w(u v), s:=s(v), t:=t(v)$, and consider the sequence of five vertices $u \prec s \prec w \prec v \prec t$.

Let $D$ be the disk supporting $D_{\sigma}(u, v)$, and let $D^{\prime}$ be the disk supporting $D_{\sigma}(s, t)$, see Fig. 4c. By our observation above, the only vertex in the range $u \preceq x \preceq v$ not in $D$ is $w$, and the only vertex in the range $s \preceq x \preceq t$ not in $D^{\prime}$ is $v$. In particular, we have $s \in D, w \notin D, w \in D^{\prime}$, and $v \notin D^{\prime}$, see Fig. $4 \mathrm{c}$.

Let $\ell$ be the directed line from $s$ to $v$. Since $\sigma<\pi / 2$, the center of $D$ lies to the left of the oriented line $u v$, and therefore to the left of $\ell$. Similarly, the center of $D^{\prime}$ lies to the left of $s t$, and therefore to the left of $\ell$. Since $s \in D$ and $v \notin D^{\prime}$, we have $D^{\prime} \cap \ell \subset D \cap \ell$. Furthermore, since $s \prec w \prec v$, the point $w$ lies to the right of $\ell$, and in $D^{\prime} \backslash D$. 
By Lemma 6 the radius of $D^{\prime}$ is therefore less than the radius of $D$. Since $D_{\sigma}(u, v)$ and $D_{\sigma}(s, t)$ are affinely similar, this implies that $d(s, t)<d(u, v)$. This, however, is a contradiction to our choice of $v$, and our assumption $n>k+1$ is false.

A limit argument now gives the following theorem:

Theorem 1 For any compact convex set $C \subset \mathbb{R}^{2}$ and any integer $k>2$ there is a convex $k$-gon $Q$ contained in $C$ such that $\alpha(C, Q) \geq \alpha_{k}$, where $\alpha_{k}=(1-2 /(k+1)) \pi$. This bound is best possible.

Proof We start by proving the theorem for the special case when $C$ is a convex polygon. Among all subpolygons $R$ of $C$ with $\varphi_{k}(R) \geq \varphi_{k}(C)$, let $P$ be one with the minimal number of vertices. This implies that for every proper subpolygon $R$ of $P$ we have $\varphi_{k}(R)<\varphi_{k}(C) \leq \varphi_{k}(P)$, and so $P$ is worst-approximable. By Lemma $7, P$ is a $(k+1)$-gon. It follows that $P$ has at least one interior angle that is at least $\alpha_{k}$. Choosing $Q$ to be the convex hull of the remaining $k$ vertices gives $\varphi(P, Q) \leq \pi-\alpha_{k}$, and so $\varphi_{k}(C) \leq \varphi_{k}(P) \leq \pi-\alpha_{k}$, proving the theorem.

Next we consider a general compact convex figure $C$ in the plane. We choose a sequence $P_{i}$ of convex polygons inscribed within $C$ that converges to $C$ with respect to the Hausdorff-distance. For each $P_{i}$ there is a subpolygon $Q_{i} \subset P_{i}$ with $k$ vertices and $\alpha\left(P_{i}, Q_{i}\right) \geq \alpha_{k}$.

We interpret the $k$ vertices of $Q_{i}$ as a point $q_{i} \in \mathbb{R}^{2 k}$. Since $Q_{i} \subset C$, this sequence is bounded, and so the Bolzano-Weierstrass theorem guarantees the existence of a subsequence that converges to a point $q \in \mathbb{R}^{2 k}$. We interpret $q$ again as a $k$-vertex polygon $Q$. It is easy to see that $Q$ is a convex polygon with at most $k$ vertices.

It remains to show that $\alpha(C, Q) \geq \alpha_{k}$. Let $p \in C \backslash Q$. There is a sequence of points $p_{i} \in P_{i}$ with $\lim _{i \rightarrow \infty} p_{i}=p$. Since $\alpha\left(P_{i}, Q_{i}\right) \geq \alpha_{k}$, that implies that there are vertices $x_{i}, y_{i}$ of $Q_{i}$ such that $\angle x_{i} p_{i} y_{i} \geq \alpha_{k}$. We consider the sequence $\left(x_{i}, y_{i}\right)$ in $\mathbb{R}^{4}$ and apply again the Bolzano-Weierstrass theorem. We pass to a subsequence where $x:=\lim _{i \rightarrow \infty} x_{i}$ and $y:=\lim _{i \rightarrow \infty} y_{i}$ exist. The points $x$ and $y$ are necessarily vertices of $Q$. The angle $\angle x p y$ is a continuous function in $(x, y, p)$ as long as the three points remain distinct. Since $p \notin Q$, this implies $\angle x p y \geq \alpha_{k}$, and the theorem follows.

The regular $(k+1)$-gon shows that the bound is indeed best possible.

Theorem 1 implies that $\alpha(k)=\alpha_{k}$, positively answering Conjecture 1 (the case $k=2$ was already known to be true).

\section{Hausdorff-Approximation}

In this section we consider the case of Hausdorff approximation, and our error function $\psi$ is the distance between $v$ and the segment $s t$, as illustrated in Fig. 1b. We continue with the analysis of worst-approximable polygons of Sect. 3: $k>2$ and $\sigma>0$ are fixed, and we consider a convex $n$-gon $P$ with $\varphi_{k}(P)>\sigma$, but such that for every proper subpolygon $R$ of $P$ we have $\varphi_{k}(R) \leq \sigma$.

We need a small geometric result similar to Lemma 2: 
(a)

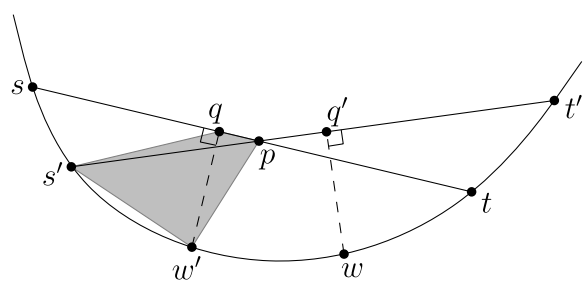

(b)

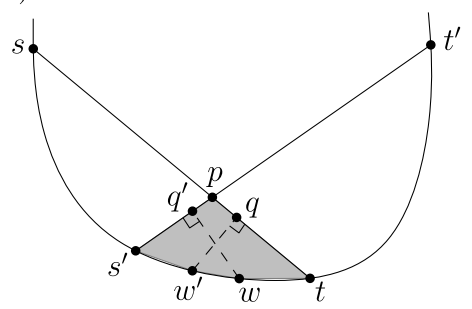

Fig. 5 Proof of Lemma 8

Lemma 8 With respect to the Hausdorff-approximation, it is impossible for two bases $s t$ and $s^{\prime} t^{\prime}$ to realize the order $s \prec s^{\prime} \prec w\left(s^{\prime} t^{\prime}\right) \prec w(s t) \prec t \prec t^{\prime}$.

Proof Suppose that there exist two bases $s t$ and $s^{\prime} t^{\prime}$ such that $s \prec s^{\prime} \prec w\left(s^{\prime} t^{\prime}\right) \prec$ $w(s t) \prec t \prec t^{\prime}$. Let $w:=w(s t)$, let $w^{\prime}:=w\left(s^{\prime} t^{\prime}\right)$, let $q$ be the point on the segment $s t$ minimizing the distance $d\left(w^{\prime}, q\right)$, and let $q^{\prime}$ be the point on $s^{\prime} t^{\prime}$ minimizing $d\left(w, q^{\prime}\right)$.

The bases $s t$ and $s^{\prime} t^{\prime}$ must intersect in a point $p$ lying in $P$. We first assume that $q$ lies on the segment $s p$, see Fig. 5a. Since $s s^{\prime} w^{\prime} t$ is a convex quadrilateral, so is $p q s^{\prime} w^{\prime}$. Its diagonals $s^{\prime} p$ and $w^{\prime} q$ intersect, implying $d\left(w^{\prime}, s^{\prime} t^{\prime}\right)<d\left(w^{\prime}, q\right) \leq \sigma$, a contradiction to $w^{\prime}=w\left(s^{\prime} t^{\prime}\right)$. It follows that $q$ must lie on the segment $p t$, and by symmetry $q^{\prime}$ lies on $s^{\prime} p$.

Since $p s^{\prime} w^{\prime} w t$ is a convex pentagon (the intersection of the two subpolygons $s s^{\prime} w^{\prime} w t$ and $s^{\prime} w^{\prime} w t t^{\prime}$ of $P$ ), $q$ lies on its edge $p t$, and $q^{\prime}$ lies on its edge $s^{\prime} p$, the chain $q^{\prime} w^{\prime} w q$ is a convex quadrilateral, see Fig. 5b. The sum of the lengths of its diagonals $q^{\prime} w$ and $q w^{\prime}$ must be larger than the sum of the lengths of the opposite sides $q^{\prime} w^{\prime}$ and $q w$. This, however, is a contradiction to $d\left(w, q^{\prime}\right) \leq \sigma<d(w, q)$ and $d\left(w^{\prime}, q\right) \leq \sigma<d\left(w^{\prime}, q^{\prime}\right)$, and the lemma follows.

Lemma 9 There is an integer $0<r \leq m$ such that for every $v_{i}$ we have $s\left(v_{i}\right)=v_{i-r}$.

Proof Let $r$ be the smallest integer $>0$ such that there is a vertex $v_{i}$ with $s\left(v_{i}\right)=$ $v_{i-r}$. We will show that then $s\left(v_{i+1}\right)=v_{i-r+1}$, and by induction this implies the lemma. Assume the opposite, that is, $s\left(v_{i+1}\right) \neq v_{i-r+1}$. By definition of $r$, we cannot have $v_{i-r+1} \prec s\left(v_{i+1}\right) \prec v_{i+1}$, and therefore $s\left(v_{i+1}\right) \prec s\left(v_{i}\right) \prec v_{i} \prec v_{i+1} \prec$ $t\left(v_{i+1}\right) \prec t\left(v_{i}\right)$, which is a contradiction to Lemma 8 .

Note that the above lemma also implies $t\left(v_{i}\right)=v_{i-r+m+1}$. From now on, let $r$ be as in Lemma 9. We may assume $r \leq m / 2$, otherwise we can work with the mirror image of $P$.

The rest of the proof is similar in spirit to Lemma 7, but is technically more complicated.

Lemma 10 We have $3 r>m+1$. 
Proof Assume $3 r \leq m+1$, and let $v_{i}$ be a vertex minimizing the Euclidean distance $d\left(v_{i}, v_{i-r}\right)$. We concentrate on the vertices

$$
\begin{aligned}
& s_{1}=v_{i-2 r}, \quad s_{2}=v_{i-r}, \quad s_{3}=v_{i}, \quad s_{4}=v_{i+r}, \\
& t_{1}=v_{i-2 r+m+1}, \quad t_{2}=v_{i-r+m+1}, \quad t_{3}=v_{i+m+1} .
\end{aligned}
$$

Since $3 r \leq m+1$, we have $s_{1} \prec s_{2} \prec s_{3} \prec s_{4} \preceq t_{1} \prec t_{2}$. We have $s_{2}=w\left(s_{1} t_{1}\right)$, $s_{3}=w\left(s_{2} t_{2}\right)$, and $s_{4}=w\left(s_{3} t_{3}\right)$. By our choice of $v_{i}$, we have $d\left(s_{2}, s_{3}\right) \leq d\left(s_{1}, s_{2}\right)$ and $d\left(s_{2}, s_{3}\right) \leq d\left(s_{3}, s_{4}\right)$. We will show that this is impossible, implying that the assumption $3 r \leq m+1$ is false.

By rotating $P$, we can assume that the line $s_{1} t_{1}$ is horizontal. Let $S_{1}$ be the horizontal strip of width $\sigma$ bounded from above by $s_{1} t_{1}$, and let $S_{2}$ be the strip of width $\sigma$ to the right of the oriented line from $s_{2}$ to $t_{2}$. The intersection $R=S_{1} \cap S_{2}$ is a rhombus. Its top-left corner $p_{1}$ is the intersection point of the segments $s_{1} t_{1}$ and $s_{2} t_{2}$. We denote the remaining corners counter-clockwise as $p_{2}, p_{3}$, and $p_{4}$, see Fig. 6. Since $s_{1} \prec s_{3} \prec s_{4} \preceq t_{1}$, we have $s_{3}, s_{4}, t_{1} \in S_{1}$. Since we also have $s_{4}, t_{1} \in S_{2}$, it follows that $s_{4}, t_{1} \in R$.

We now first show that $s_{4}$ must lie strictly right of $s_{3}$. If $t_{1}$ lies right of $s_{3}$, this follows from the fact that $s_{4}$ lies strictly to the right of the oriented line $s_{3} t_{1}$ and to the left of the oriented line $s_{1} s_{3}$. If $t_{1}$ lies left of $s_{3}$, then $d\left(s_{3}, s_{1} t_{1}\right) \leq \sigma$ implies $d\left(s_{3}, t_{1}\right) \leq \sigma$. If $s_{4}$ lies not strictly right of $s_{3}$, then the angle $\angle s_{3} s_{4} t_{1}$ is right or obtuse, and so $d\left(s_{3}, s_{4}\right) \leq d\left(s_{3}, t_{1}\right) \leq \sigma$, a contradiction to $s_{4}=w\left(s_{3} t_{3}\right)$.

We will now first consider the case that the angle $\angle s_{2} p_{1} t_{1}$ is at least $90^{\circ}$, so $R$ has obtuse angles at $p_{1}$ and $p_{3}$ and acute angles at $p_{2}$ and $p_{4}$, see Fig. 6a. Let $z$ be the point on the segment $p_{1} p_{2}$ at distance $\sigma$ from $p_{1}$. Since $s_{2}=w\left(s_{1} t_{1}\right), s_{2}$ must lie below $z$, and so the segment $z p_{1}$ is a subset of $s_{2} t_{2}$. Since $s_{3}$ lies in $S_{1}$ but $d\left(s_{3}, s_{2} t_{2}\right)>\sigma, s_{3}$ must lie strictly to the right of $p_{3}$. Since $s_{4} \in R$ and strictly right of $s_{3}$, this implies that $s_{3}$ lies strictly left of $p_{4}$. Since $s_{3}$ lies strictly in between the vertical lines through $p_{3}$ and $p_{4}$, and $s_{4}$ lies in between the vertical lines through $s_{3}$ and $p_{4}$, we have $d\left(s_{3}, s_{4}\right)<d\left(p_{3}, p_{4}\right)$.

Consider now the point $s_{2}$. We argue that it must lie strictly below $p_{2}$. Indeed, otherwise it lies inside $S_{1}$ on the segment $p_{1} p_{2}$. Since $d\left(s_{2}, s_{1} t_{1}\right)>\sigma$, this implies that
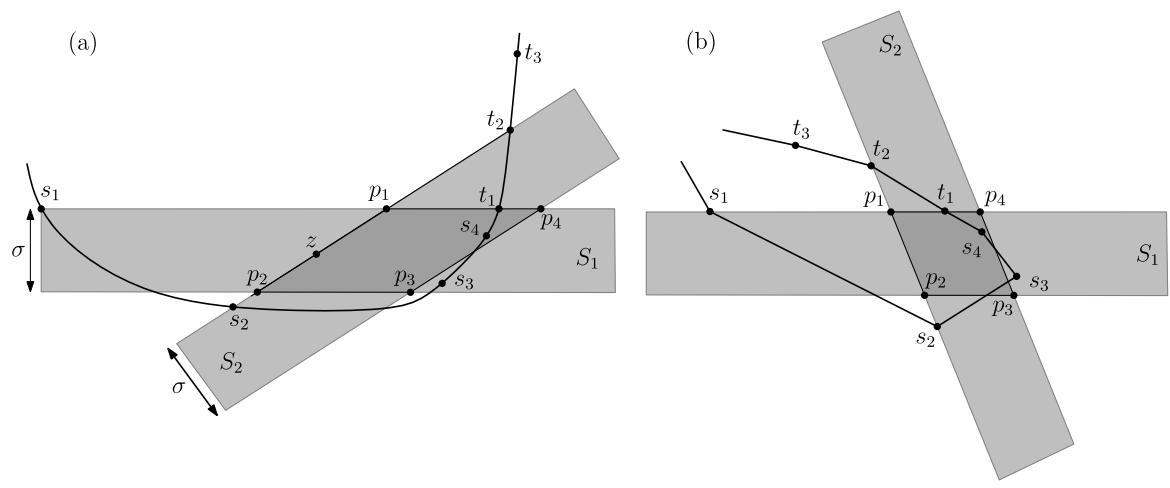

Fig. 6 Proof of Lemma 10 
$s_{1}$ lies right of $s_{2}$. Then the angle $\angle s_{2} s_{1} p_{1}$ is obtuse, implying $d\left(s_{2}, s_{1}\right) \leq d\left(s_{2}, p_{1}\right)$. But since $s_{3}$ lies strictly right of $p_{3}$, we have $d\left(s_{2}, s_{3}\right)>d\left(s_{2}, p_{1}\right) \geq d\left(s_{2}, s_{1}\right)$, a contradiction to our choice of $s_{3}=v_{i}$.

Since $s_{2}$ lies strictly below $p_{2}$ on the line $p_{1} p_{2}$ and $s_{3}$ lies in $S_{1}$ right of $p_{3}$, we have $d\left(s_{2}, s_{3}\right)>d\left(p_{2}, p_{3}\right)=d\left(p_{3}, p_{4}\right)>d\left(s_{3}, s_{4}\right)$, again a contradiction to our choice of $s_{3}=v_{i}$.

It remains to consider the case that the angle $\angle s_{2} p_{1} t_{1}$ is less than $90^{\circ}$, so $R$ has acute angles at $p_{1}$ and $p_{3}$ and obtuse angles at $p_{2}$ and $p_{4}$, see Fig. 6b. If $s_{3}$ does not lie in $R$, we immediately have a contradiction: Since $s_{4}$ must lie to the left of the oriented line $t_{1} s_{3}$ and right of $s_{3}$, it cannot lie in $R$. If $s_{3}$ lies in $R$, the nearest point to $s_{3}$ on the segment $s_{2} t_{2}$ must be $s_{2}$, as otherwise $d\left(s_{3}, s_{2} t_{2}\right) \leq \sigma$. This implies that $s_{3}$ lies below the line $\ell$ through $s_{2}$ orthogonal to $p_{1} p_{2}$, and outside the circle $T$ with radius $\sigma$ around $s_{2}$.

Since the segments $s_{2} s_{4}$ and $s_{3} t_{3}$ intersect and $s_{4}=w\left(s_{3} t_{3}\right)$, we must have $d\left(s_{2}, s_{4}\right)>\sigma$. This implies that the nearest point to $s_{4}$ on $s_{2} t_{2}$ must be different from $s_{2}$, and so $s_{4}$ must lie above the line $\ell$.

Now we observe that if $s_{3}$ lies above $s_{2}$, then it lies strictly right of the intersection point of $\ell$ and the line $p_{4} p_{3}$. Since $s_{4}$ lies right of $s_{3}$, this implies that $s_{4}$ cannot lie in $R$ above $\ell$, a contradiction, and so $s_{3}$ must lie below $s_{2}$. Since $s_{3} \in R$, this implies $s_{2} \in R$. Therefore, the point nearest to $s_{2}$ on $s_{1} t_{1}$ must be $t_{1}$, and $t_{1}$ must lie left of $s_{2}$. This implies that the angle $\angle t_{1} s_{2} s_{3}$ is obtuse, and so $d\left(t_{1}, s_{3}\right)>d\left(t_{1}, s_{2}\right)>\sigma$. Since $s_{3}$ lies right of $t_{1}$, this implies $d\left(s_{3}, s_{1} t_{1}\right)>\sigma$, a contradiction.

Theorem 2 If $P$ is a convex $n$-gon that is worst-approximable with respect to the Hausdorff-distance and $k>2$ then $n=k+1$.

Proof We assume $n>k+1$ and set $\rho:=m+1-r$. Since $r \leq m / 2$, we have $2 \rho>$ $m+1$. Let $R$ be the subpolygon of $P$ formed by the vertices $v_{0}, v_{\rho}, v_{2 \rho}, \ldots, v_{\ell \rho}$, where $\ell$ is an integer such that $(\ell+1) \rho \leq k m+1<(\ell+2) \rho$. Since $\rho>1, R$ is a proper subpolygon of $P$. We will show that $\varphi_{k}(R)>\sigma$, a contradiction to the assumption that $P$ is worst-approximable.

We first show that $\ell \geq k$. We assume the contrary, that is $\ell<k$. This implies $\ell+2 \leq k+1$. Since $k m+1<(\ell+2) \rho$, we have $k m+2 \leq(\ell+2) \rho$. By Lemma 10 we have $3 r>m+1$, which implies $3 r \geq m+2$, and therefore $\rho \leq \frac{2}{3} m+\frac{1}{3}$. This gives

$$
3 k m+6=3(k m+2) \leq 3(\ell+2) \rho \leq 3(k+1)\left(\frac{2}{3} m+\frac{1}{3}\right)=2 k m+2 m+k+1 .
$$

Rearranging the terms gives $k m-2 m-k+5 \leq 0$ or $(k-2)(n-1) \leq-3$, a contradiction with $k>2$ and $m \geq 1$. It follows that our claim holds, that is $\ell \geq k$.

It remains to prove that $\varphi_{k}(R)>\sigma$. Note that $R$ has at least $k+1$ vertices, and so a $k$-vertex subpolygon $Q$ of $R$ must exclude at least one vertex of $R$. We have three cases (recall that arithmetic on the indices of $v$ is modulo $n$ ):

- $v_{i \rho}$ is excluded, for $0<i<\ell$.

By Lemma 9, $v_{i \rho}$ is the witness of the base $\left(v_{(i+1) \rho-(m+1)}, v_{(i+1) \rho}\right)$. Since $2 \rho>$ $m+1, v_{(i-1) \rho} \prec v_{(i+1) \rho-(m+1)} \prec v_{i \rho} \prec v_{(i+1) \rho}$, and so monotonicity of $\psi$ implies that $\psi\left(v_{i \rho}, v_{(i-1) \rho}, v_{(i+1) \rho}\right)>\sigma$. 
- $v_{0}$ is excluded.

$v_{0}$ is the witness of the base $\left(v_{\rho-(m+1)}, v_{\rho}\right)$. Since $(\ell+1) \rho \leq k m+1=n$ and $2 \rho>$ $m+1$, we have $\ell \rho \leq n-\rho<n+\rho-(m+1)$, which implies $v_{\ell \rho} \prec v_{\rho-(m+1)} \prec$ $v_{0} \prec v_{\rho}$, and so $\psi\left(v_{0}, v_{\ell \rho}, v_{\rho}\right)>\sigma$.

- $v_{\ell \rho}$ is excluded.

$v_{\ell \rho}$ is the witness of the base $\left(v_{(\ell+1) \rho-(m+1)} v_{(\ell+1) \rho}\right)$. Since $2 \rho>m+1$ and $(\ell+$

1) $\rho \leq n$, we have $v_{(\ell-1) \rho} \prec v_{(\ell+1) \rho-(m+1)} \preceq v_{0}$, and so $\psi\left(v_{\ell \rho}, v_{(\ell-1) \rho}, v_{0}\right)>\sigma$.

In all cases, $\varphi(R, Q)>\sigma$, and so $\varphi_{k}(R)>\sigma$.

The following approximation result is a direct application of Theorem 2.

Theorem 3 For any convex polygon $P$ of perimeter at most one and any $k>2$ there exists a subpolygon $Q$ of $P$ with $k$ vertices such that $\varphi(P, Q) \leq \frac{1}{k+1} \sin \frac{\pi}{k+1}$ with respect to the Hausdorff-distance. If $P$ is a regular $(k+1)$-gon, this bound is best possible.

Proof Let $R$ be a subpolygon of $P$ with the smallest number of vertices such that $\varphi_{k}(R) \geq \varphi_{k}(P)$. Then $R$ is worst-approximable and, by Theorem $2, R$ is a $(k+1)$ gon. The following lemma now implies the theorem.

Lemma 11 Let $k>2$ and let $P$ be a convex $(k+1)$-gon with $\varphi_{k}(P)=1$ with respect to the Hausdorff-distance. Then the perimeter of $P$ is at least $(k+1) / \sin (\pi /(k+1))$, and this bound is tight for the regular $(k+1)$-gon.

Proof We set $n=k+1$, and let $P$ be a convex $n$-gon with $\varphi_{k}(P)=1$ of minimal perimeter (the existence of such a $P$ follows from a compactness argument). Let $v_{1}, \ldots, v_{n}$ denote the vertices of $P$ in counter-clockwise order, and let $Q_{i}$ be the subpolygon excluding the vertex $v_{i}$. We first argue that $\varphi\left(P, Q_{i}\right)=1$ for all $i$. Indeed, if there is a vertex $v_{i}$ such that $\varphi\left(P, Q_{i}\right)>1$, then we can move $v_{i}$ slightly along the directed line from $v_{i}$ to $v_{i+2}$. This decreases the perimeter while keeping $\varphi_{k}(P)=1$, a contradiction to the choice of $P$.

Let $\gamma_{i}$ be the angle made by the oriented diagonals $v_{i-1} v_{i+1}$ and $v_{i} v_{i+2}$. Since the direction of the diagonal $v_{i-1} v_{i+1}$ is a tangent direction at $v_{i}$, we have

$$
\sum_{i=1}^{n} \gamma_{i}=2 \pi
$$

The distance of $v_{i}$ from the line $v_{i-1} v_{i+1}$ and the distance of $v_{i+1}$ from the line $v_{i} v_{i+2}$ is one. This implies that the length of the edge $v_{i} v_{i+1}$ is $1 / \sin \left(\gamma_{i} / 2\right)$, see Fig. 7. We set $x_{i}=\gamma_{i} / 2$, and define $f(x)=1 / \sin x$. We then have

$$
\begin{gathered}
\sum_{i=1}^{n} x_{i}=\pi, \\
\operatorname{peri}(R)=\sum_{i=1}^{n} f\left(x_{i}\right) .
\end{gathered}
$$


Fig. 7 Proof of Lemma 11

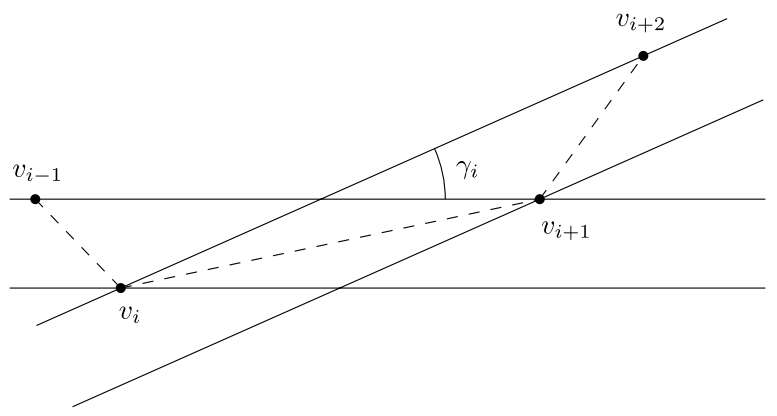

Since $f^{\prime \prime}(x)>0$ for $0<x<\pi / 2$, the function $f(x)$ is convex on the interval $(0, \pi / 2)$. Since $0<x_{i}<\pi / 2$, we can thus apply Jensen's inequality to give us

$$
f\left(\frac{\sum_{i} x_{i}}{n}\right) \leq \frac{\sum_{i} f\left(x_{i}\right)}{n}=\frac{\operatorname{peri}(R)}{n} .
$$

Multiplying by $n$ gives peri $(R) \geq n f(\pi / n)=n / \sin (\pi / n)$, completing the proof.

\section{Conclusions}

We showed that worst-approximable polygons are $(k+1)$-gons for both the Hausdorff-distance and the aperture-angle distance. A large part of the argument is purely combinatorial, using only the monotonicity of the distance function $\psi$. To finish the argument, however, we needed to make use of some geometric properties of the two distance functions we considered; in Lemma 7 for the aperture angle, and in Lemmas 8 and 10 for the Hausdorff distance. We must ask: are we just blinded by the geometry to overlook an entirely combinatorial proof that would apply for any monotone distance function?

We gave one application of Theorem 2, but it's worth pointing out that the theorem is really far more general, and applies to many families $\mathcal{P}$ of convex polygons. In many cases the regular $(k+1)$-gon appears to be the worst case, but this is not always the case, for instance, because the family $\mathcal{P}$ does not contain it. (Consider, for instance, the family of all convex polygons with vertices on a given ellipse.)

Acknowledgements We thank Sándor Fekete for the historical background of the aperture angle problem, and Peter Brass for pointing out the similarity in the statement of the two conjectures as well as his help in accessing the literature. We thank Peter Brass, Hyeon-Suk Na, and Chan-Su Shin for helpful discussions during a mini-workshop at Soongsil University. Finally, we thank Mira Lee for suggesting the approach used in the proof of Theorem 3.

\section{References}

1. Bose, P., Hurtado, F., Omaña-Pulido, E., Snoeyink, J., Toussaint, G.T.: Some aperture angle optimization problems. Algorithmica 33, 411-435 (2002)

2. Brass, P.: On the approximation of polygons by subpolygons. In: Proc. European Workshop Comput. Geom. (EuroCG), pp. 59-61 (2000) 
3. Brass, P., Lassak, M.: Problems on approximation by triangles. Geombinatorics 10, 103-115 (2001)

4. Brass, P., Moser, W., Pach, J.: Research Problems in Discrete Geometry. Springer, Berlin (2005)

5. Cheong, O., van Oostrum, R.: Reaching a polygon with directional uncertainty. Int. J. Comput. Geom. Appl. 11, 197-214 (2001)

6. Fekete, S.: Personal communication

7. Hoffmann, F., Icking, C., Klein, R., Kriegel, K.: Moving an angle around a region. In: Algorithm Theory-SWAT'98. Lecture Notes in Computer Science, vol. 1432, pp. 71-82 (1998)

8. Jenkner, M.: Approximation konvexer Kurven. Master's thesis, Universität zu Köln (1997)

9. Popov, V.A.: Approximation of convex bodies. C. R. Acad. Bulg. Sci. 21, 993-995 (1968) (in Russian, see Zbl 215.50601)

10. Popov, V.A.: Approximation of convex sets. B'lgar. Akad. Nauk. Otdel. Mat. Fiz. Nauk. Izv. Mat. Inst. 11, 67-80 (1970) (in Bulgarian, with Russian and English summary) 